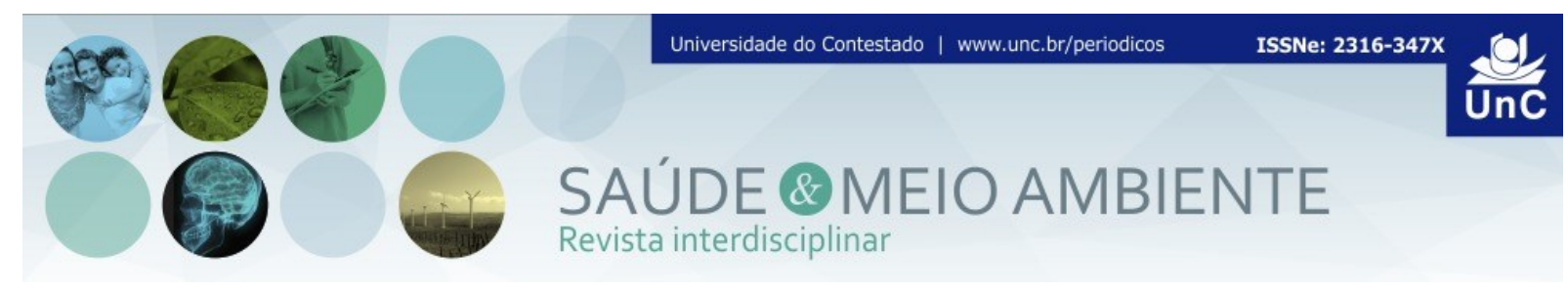

\title{
ESTUDO COMPARATIVO DE ESQUEMAS TERAPÊUTICOS PARA TUBERCULOSE A PARTIR DE DADOS DO SERVIÇO MUNICIPAL DE INFECTOLOGIA DE CAXIAS DO SUL
}

\author{
Francie Bueno ${ }^{1}$ \\ Tathiane Andréa Klanovicz ${ }^{2}$ \\ Luciana Mello de Oliveira ${ }^{3}$ \\ Claudia Wollhein ${ }^{4}$ \\ Patricia Regina Araujo ${ }^{5}$ \\ Tarcio Jovani Laurini ${ }^{6}$
}

\begin{abstract}
RESUMO
Os objetivos do presente estudo foram avaliar o tratamento com tuberculostáticos para verificar o índice de abandono nos esquemas terapêuticos $(2 \mathrm{RHZ} / 4 \mathrm{RH}$ e $2 \mathrm{RHZE} / 4 \mathrm{RH}$ ), e identificar fatores preditivos ao abandono do tratamento. Foram utilizados dados de literatura e aqueles disponibilizados pelo Serviço Municipal de Infectologia de Caxias do Sul no período de 2011 a 2013. O teste qui-quadrado de Pearson ou o teste exato de Fisher foram utilizados para a comparação de associação, entre o desfecho do tratamento e as variáveis. Os esquemas terapêuticos avaliados nesse estudo não atingiram as metas da OMS para o controle da tuberculose. Os fatores preditivos do abandono ao tratamento foram: sexo masculino, faixa etária entre 20-39 anos, baixa escolaridade, caso novo e presença de comorbidades. Desses fatores os que apresentaram significância estatística associados ao desfecho do tratamento foram o ingresso (caso novo) e a presença de comorbidades. Para se atingir o controle da tuberculose é necessário a diminuição dos índices de abandono ao tratamento.
\end{abstract}

Palavras-chave: Tuberculose. Abandono do tratamento. 2RHZ/4RH. 2RHZE/4RH

\footnotetext{
${ }^{1}$ Centro de Ciências biológicas e da Saúde, Curso de Farmácia. Universidade de Caxias do Sul. Rio Grande do Sul. Brasil. E-mail: franciebueno@yahoo.com.br

${ }^{2}$ Centro de Ciências biológicas e da Saúde, Curso de Farmácia. Universidade de Caxias do Sul. Rio Grande do Sul. Brasil. E-mail: tathi0609@hotmail.com

${ }^{3}$ Curso de Farmácia. Pontifícia Universidade Católica do Rio Grande do Sul. Rio Grande do Sul. Brasil. E-mail: oliveiralm@gmail.com

${ }^{4}$ Centro de Ciências biológicas e da Saúde, Curso de Farmácia. Universidade de Caxias do Sul. Rio Grande do Sul. Brasil. E-mail: cwollhei@ucs.br

${ }^{5}$ Centro de Ciências biológicas e da Saúde, Curso de Farmácia. Universidade de Caxias do Sul. Rio Grande do Sul. Brasil. E-mail: praraujo@ucs.br

${ }^{6}$ Secretaria Municipal de Saúde, Prefeitura de Caxias do Sul. Rio Grande do Sul. Brasil. E-mail: tlaurini@caxias.rs.gov.br
} 


\title{
COMPARATIVE STUDY OF THERAPEUTIC PROGRAMMES FOR TUBERCULOSIS, FROM DATA FROM THE PUBLIC DEPARTMENT OF INFECTIOUS DISEASES OF THE CITY OF CAXIAS DO SUL
}

\begin{abstract}
The aims of the present study were to assess treatment with tuberculostatics to verify the drop out rate in therapeutic programmes (2RHZ/4RH and $2 \mathrm{RHZE} / 4 \mathrm{RH})$, and to identify predictive factors associated to drop out of treatment. For this purpose, data gathered from literature and those made available by the local Public Department of Infectious Diseases of the city of Caxias do Sul in the 2011-2013 period were used. Pearson's chi-squared test or Fisher's exact test have been used to compare and associate the outcome of the treatment and the variables. The therapeutic programmes assessed in this study did not meet the WHO targets for tuberculosis control. The predictive factors for dropping out of treatment were: male, age between 20-39 years old, low level of education, new case, and presence of comorbidities. Out of these factors, the ones that resulted statistically significant when associated to the outcome of the treatment were admission (new case) and presence of comorbidities. In order to achieve effective tuberculosis control, it is necessary to reduce the factors of treatment drop out.
\end{abstract}

Keyword: Tuberculosis. Drop out of treatment. 2RHZ/4RH. 2RHZE/4RH.

\section{INTRODUÇÃO}

A tuberculose é uma doença infectocontagiosa causada pelo Mycobacterium tuberculosis (DUCATI; BASSO; SANTOS, 2008). A transmissão se dá por via respiratória, pela inalação de aerossóis produzidos pela tosse, fala ou espirro de um doente com tuberculose ativa de vias aéreas (BRASIL, 2011c).

Esta doença atinge indiscriminadamente indivíduos aparentemente saudáveis e pode ser muito grave em indivíduos imunocomprometidos. Trata-se de uma doença primariamente dos pulmões, mas pode disseminar-se para outros locais do organismo ou evoluir para uma infecção generalizada, conhecida como tuberculose miliar (MINS et al, 1999; SANTOS, 2010). Os sintomas clássicos da tuberculose pulmonar são: tosse persistente por tempo igual ou superior a três semanas, produtiva ou não (commuco e eventualmente sangue), febre vespertina, sudorese

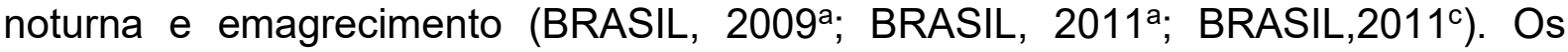
exames utilizados para o diagnóstico de certeza são a baciloscopia do escarro e a cultura do escarro ou outros líquidos. Já os exames utilizados como auxiliares para o diagnóstico são a radiografia de tórax, o teste tuberculínico (PPD), que evidencia o contato prévio com o bacilo (BRASIL, 2009a; BRASIL, 2011a; BRASIL, 2011c; SOUZA, VASCONCELOS, 2005).

A importância epidemiológica da tuberculose se traduz em dados veiculados pela Organização Mundial da Saúde (OMS) mostrando que existem dois bilhões de 
infectados pelo $M$. tuberculosisna população mundial, propiciando a ocorrência de oito milhões de casos novos/ano e a morte de aproximadamente dois milhões de pessoas (HIJJAR et al, 2011; WHO, 2011).

Quando a OMS decretou esta doença em estado de emergência mundial, em 1993, estava fundamentada pelos altos índices de incidência e mortalidade existentes, principalmente em países com piores condições socioeconômicas. Naquele momento, era uma doença negligenciada, sem prioridade no seu combate, mas com surgimento de surtos multirresistentes até em países de primeiro mundo, como nos Estados Unidos, passou a haver mobilização para o controle dessa doença (DUCATI; BASSO; SANTOS, 2008; HIJJAR et al., 2011).

As metas do milênio para a tuberculose, pactuadas pela Organização das Nações Unidas (ONU) visam reduzir a incidência e de mortalidade em $50 \%$ até 2015, comparados aos valores de 1990, e eliminar a tuberculose como problema de saúde pública até 2050. Apesar do Brasil ser ainda um dos 22 países responsáveis por $90 \%$ dos casos de tuberculose do mundo, até o ano de 2007 , ocorreu em nosso país uma queda de $26 \%$ na incidência e de $32 \%$ na mortalidade por tuberculose (BRASIL, 2011 b; BRASIL, 2012; SBPT, 2009)

Dados do Ministério da Saúde (BRASIL, 2010) revelam que o Rio Grande do Sul está na $6^{a}$ posição em relação a outros estados brasileiros, com uma incidência de 42,5 para cada 100 mil habitantes, sendo Porto Alegre a primeira capital do Brasil, Região Sul e Fronteira Oeste as regiões de maior incidência da doença (PAIVA; PERERIRA; MOREIRA, 2011).

A estratégia de controle da tuberculose é feita por programas governamentais, que consistem em diagnosticar e tratar os casos o mais rapidamente possível a fim de evitar a difusão da doença que se propaga rapidamente. Na última norma técnica, publicada em outubro de 2009 , o tratamento indicado para todos os casos novos de tuberculose pulmonar e extrapulmonar, assim como para todos os casos de recidiva e de retorno após abandono, é a utilização de quatro fármacos em um único comprimido com dose fixa combinada: rifampicina, isoniazida, pirazinamida e etambutol por dois meses e, numa segunda fase, isoniazida e rifampicina por mais quatro meses (esquema $2 \mathrm{RHZE} / 4 \mathrm{RH}$ ), para pacientes adolescentes e adultos. Para crianças de até 10 anos permanece a recomendação de tratamento inicialmente padronizadocom rifampicina, isoniazida e pirazinamida, na primeira fase e, rifampicina e isoniazida na segunda fase (2RHZ/4RH) (BRASIL, 2009b; BRASIL, 2011'c; SBPT, 2009).

Um dos maiores problemas no tratamento da tuberculose $\mathrm{e}$ consequentemente de saúde pública é a adesão aos esquemas de tratamento até o fim, sem interrupção, uma vez que isso pode ocasionar falência do tratamento, podendo levar a uma recidiva da doença e aumentar a possibilidade de transmissão, ou até mesmo de resistência aos antimicrobianos administrados, além de aumentar o tempo e o custo do tratamento. Por sua vez, o conhecimento dos fatores associados ao abandono do tratamento da tuberculose (caracterizado pelo não comparecimento do doente ao serviço de saúde por mais de trinta dias consecutivos, após a data aprazada para o retorno (SÁ, et al., 2007) é necessário 
para a identificação das barreiras que culminam para a não adesão ao tratamento (CHIRINOS; MEIRELLES, 2011; SANTOS, 2012; VILA, et al., 2008).

O presente trabalho teve por objetivo avaliar 0 tratamento com tuberculostáticos com o intuito de verificar o índice de abandono nos diferentes esquemas terapêuticos (2RHZ/4RH e 2RHZE/4RH), e identificar os fatores preditivos (sexo, faixa etária, escolaridade, ingresso e comorbidades) ao abandono do tratamento da tuberculose.

\section{METODOLOGIA}

Trata-se de um estudo descritivo de dados secundários, com abordagem quantitativa, realizado no Serviço Municipal de Infectologia que funciona junto ao Centro Especializado de Saúde (CES), do município de Caxias do Sul, RS, Brasil, e de caráter comparativo com abordagem qualitativa ao que se refere ao referencial teórico. Para a realização do estudo foram utilizados os dados secundários presentes no sistema informatizado SIS.SAP (software para a gestão da saúde no Setor Público) de pacientes em tratamento da tuberculose entre janeiro de 2011 a dezembro de 2013. Cabe salientar que estes dados foram obtidos com auxílio e supervisão do farmacêutico responsável pelo Serviço Municipal de Infectologia.

A coleta de dados incluiu as seguintes variáveis: dados demográficos (idade, gênero e escolaridade), ingresso no serviço de saúde (caso novo, recidiva, retratamento após abandono, transferência e investigação/acompanhamento) e presença de comorbidades. Além disso, foram obtidos dados como: motivo da alta do paciente e número total de pacientes em tratamento selecionados pelo ano da notificação, de acordo com o período de tempo estabelecido para o estudo.

A partir da análise das variáveis e do motivo da alta de cada paciente foram definidos os desfechos a serem avaliados (cura, abandono, óbito e mudança de diagnóstico ou transferência), verificado os fatores preditivos ao abandono do tratamento e comparado $\mathrm{O}$ índice de abandono nos diferentes esquemas terapêuticos: $2 \mathrm{RHZ} / 4 \mathrm{RH}$ e $2 \mathrm{RHZE} / 4 \mathrm{RH}$. Devido ao período de tempo estabelecido para a coleta de dados, o esquema terapêutico predominante nos dados coletados é - $2 \mathrm{RHZE} / 4 \mathrm{RH}$, já que a mudança do tratamento indicado para todos os casos novos, recidiva e retorno após abandono de tuberculose pulmonar e extrapulmonar se deu a partir de outubro de 2009. Sendo assim, para a comparação dos diferentes esquemas terapêuticos, a avaliação do esquema $2 \mathrm{RHZ} / 4 \mathrm{RH}$ foi baseada nos dados presentes na literatura.

Os dados coletados foram tabelados no Microsoft Office Excel 2007 e, posteriormente, importados para o programa IBM SPSS Statistics, versão 21. Inicialmente, a análise estatística dos dados foi realizada de maneira descritiva. Após, foi utilizado o teste qui-quadrado de Pearson ou o teste exato de Fisher para a comparação de associação entre o desfecho do tratamento (cura, abandono e óbito) 
e as variáveis (sexo, faixa etária, escolaridade, ingresso e comorbidades). O nível de significância adotado foi de $p<0,05$.

O presente estudo foi aprovado pelo Comitê de Ética em Pesquisa da Universidade de Caxias do Sul - RS em 17 de dezembro de 2015 (CAAE n 51879115.6.0000.5341), uma vez constatado o cumprimento dos requisitos éticos, tais como confidencialidade, segurança e anuência do Núcleo de Educação Permanente em Saúde (NEPS) da Secretaria da Saúde de Caxias do Sul, conforme protocolo $n^{\circ} 342 / 14$.

\section{RESULTADOS E DISCUSSÃO}

Foram avaliados dados eletrônicos de 437 pacientes em tratamento para a tuberculose com o esquema terapêutico 2RHZE/4RH, no Serviço Municipal de Infectologia, durante o período do estudo. Deste total, $293(67,0 \%)$ eram do gênero masculino e $144(33,0 \%)$ do gênero feminino. A Tabela 1 apresenta a distribuição dos pacientes de acordo com o gênero e faixa etária. As faixas etárias com menor número de casos se situam abaixo de 19 anos $(5,0 \%)$, aumentando substancialmente a partir dos 20 anos, com um pico de $24,7 \%$ no intervalo de $30-39$ anos.

Tabela 1 - Casos de tuberculose por faixa etária e gênero, no Serviço Municipal de Infectologia de Caxias do Sul, 2011 a 2013.

\begin{tabular}{lcccccc}
\hline \multicolumn{1}{r}{ Faixa etária } & \multicolumn{2}{c}{ Masculino } & \multicolumn{2}{c}{ Gênero } & \multicolumn{2}{c}{ Total } \\
& $\mathbf{n}$ & $\mathbf{\%}$ & $\mathbf{n}$ & $\mathbf{\%}$ & $\mathbf{n}$ & $\%$ \\
\hline $0-4$ & 1 & 0,3 & 0 & 0 & 1 & 0,2 \\
$5-9$ & 1 & 0,3 & 4 & 2,8 & 5 & 1,1 \\
$10-14$ & 0 & 0 & 1 & 0,7 & 1 & 0,2 \\
$15-19$ & 9 & 3,1 & 6 & 4,2 & 15 & 3,4 \\
$20-29$ & 63 & 21,5 & 25 & 17,4 & 88 & 20,1 \\
$30-39$ & 73 & 24,9 & 35 & 24,3 & 108 & 24,7 \\
$40-49$ & 60 & 20,5 & 26 & 18,1 & 86 & 19,7 \\
$50-59$ & 35 & 12,0 & 23 & 16,0 & 58 & 13,3 \\
+60 & 50 & 17,1 & 24 & 16,7 & 74 & 17,0 \\
lgnorado & 1 & 0,3 & 0 & 0 & 1 & 0,2 \\
Total & 293 & 100 & 144 & 100 & 437 & 100 \\
\hline
\end{tabular}

Verificou-se que $64,5 \%$ dos pacientes em tratamento obtiveram alta por cura, enquanto que $7,9 \%$ alta por abandono, $10,2 \%$ alta por óbito e $17,4 \%$ de transferência e mudança de diagnóstico. A Tabela 2 mostra a distribuição dos pacientes em relação ao gênero, faixa etária, escolaridade, ingresso e ausência ou presença de comorbidades para os motivos de alta por cura, abandono e óbito. $\mathrm{Na}$ análise estatística foram avaliados um total de 347 casos para os desfechos de tratamento cura, abandono e óbito, os demais casos encontravam-se em outros motivos de alta não selecionados para análise neste estudo. Dentre as variáveis 
avaliadas, nesse trabalho, as que apresentaram associação estatisticamente significativa com o desfecho do tratamento foram o ingresso (caso novo) e a presença de comorbidades $(p<0,05)$.

Tabela 2 - Casos de tuberculose segundo desfecho e sexo, faixa etária, escolaridade, ingresso e comorbidades, no Serviço Municipal de Infectologia de Caxias do Sul, 2011 a 2013.

\begin{tabular}{|c|c|c|c|c|c|c|c|}
\hline \multirow[t]{2}{*}{ Variável } & \multicolumn{2}{|c|}{$\begin{array}{c}\text { Cura } \\
N=271\end{array}$} & \multicolumn{2}{|c|}{$\begin{array}{c}\text { Abandono } \\
\mathrm{N}=33\end{array}$} & \multicolumn{2}{|c|}{$\begin{array}{l}\text { Óbito } \\
N=43\end{array}$} & \multirow[b]{2}{*}{$\mathrm{p}$} \\
\hline & $\mathrm{N}$ & $\%$ & $\mathrm{~N}$ & $\%$ & $\mathrm{~N}$ & $\%$ & \\
\hline \multicolumn{8}{|l|}{ Gênero } \\
\hline Masculino & 178 & 65,7 & 25 & 75,8 & 33 & 76,7 & \\
\hline Feminino & 93 & 34,3 & 8 & 24,2 & 10 & 23,3 & 0,213 \\
\hline \multicolumn{8}{|l|}{ Faixa etária } \\
\hline$<19$ & 17 & 6,3 & 2 & 6,1 & 0 & 0 & \\
\hline $20-39$ & 120 & 44,4 & 20 & 60,6 & 15 & 34,9 & \\
\hline $40-59$ & 90 & 33,3 & 8 & 24,2 & 15 & 34,9 & \\
\hline 60 e mais & 43 & 15,9 & 3 & 9,1 & 13 & 30,2 & 0,059 \\
\hline \multicolumn{8}{|l|}{ Escolaridade } \\
\hline Não alfabetizado & 25 & 9,2 & 3 & 9,1 & 2 & 4,7 & \\
\hline Até 8 anos de estudo & 153 & 56,5 & 22 & 66,7 & 22 & 51,2 & \\
\hline Mais de 8 anos de estudo & 93 & 34,3 & 8 & 24,2 & 19 & 44,2 & 102 \\
\hline \multicolumn{8}{|l|}{ Ingresso } \\
\hline Caso novo & 248 & 91,5 & 22 & 66,7 & 38 & 88,4 & \\
\hline Recidiva & 9 & 3,3 & 2 & 6,1 & 3 & 7,0 & \\
\hline $\begin{array}{l}\text { Retratamento após } \\
\text { abandono }\end{array}$ & 5 & 1,8 & 7 & 21,2 & 0 & 0 & 0,000 \\
\hline Outros* & 9 & 3,3 & 2 & 6,1 & 2 & 4,7 & \\
\hline \multicolumn{8}{|l|}{ Comorbidades } \\
\hline Ausência de comorbidades & 81 & 56,3 & 3 & 17,6 & 7 & 22,6 & \\
\hline Presença de comorbidades & 63 & 43,8 & 14 & 82,4 & 24 & 77,4 & 0,000 \\
\hline
\end{tabular}

No motivo de alta por cura $65,7 \%$ dos casos eram do sexo masculino, $44,4 \%$ na faixa etária de $20-39$ anos, $56,5 \%$ com até 8 anos de estudo, $91,5 \%$ eram casos novos e $56,3 \%$ não apresentaram comorbidades, dos 144 pacientes que possuíam o registro sobre presença ou ausência de comorbidades.

Dos pacientes que tiveram alta por abandono, $75,8 \%$ eram do sexo masculino, 60,6\% encontraram-se na faixa etária de $20-39$ anos e, $66,7 \%$ possuíam até 8 anos de estudo. Observou-se que $66,7 \%$ dos pacientes que ingressaram no serviço de saúde eram casos novos e $21,2 \%$ eram pacientes em retratamento após abandono. A informação sobre presença ou ausência de comorbidades foi avaliada em 17pacientes, os quais possuíam esse dado registrado, de um total de 33 pacientes que abandonaram o tratamento, e verificou-se que $82,4 \%$ dos pacientes apresentaram comorbidades (uma ou mais por paciente), sendo35,7\% usuários de drogas, $35,7 \%$ etilistas e $28,6 \%$ HIV positivos (Figura 1). 
Figura 1 - Distribuição das comorbidades em relação ao motivo da alta, no Serviço Municipal de Infectologia de Caxias do Sul, 2011 a 2013.

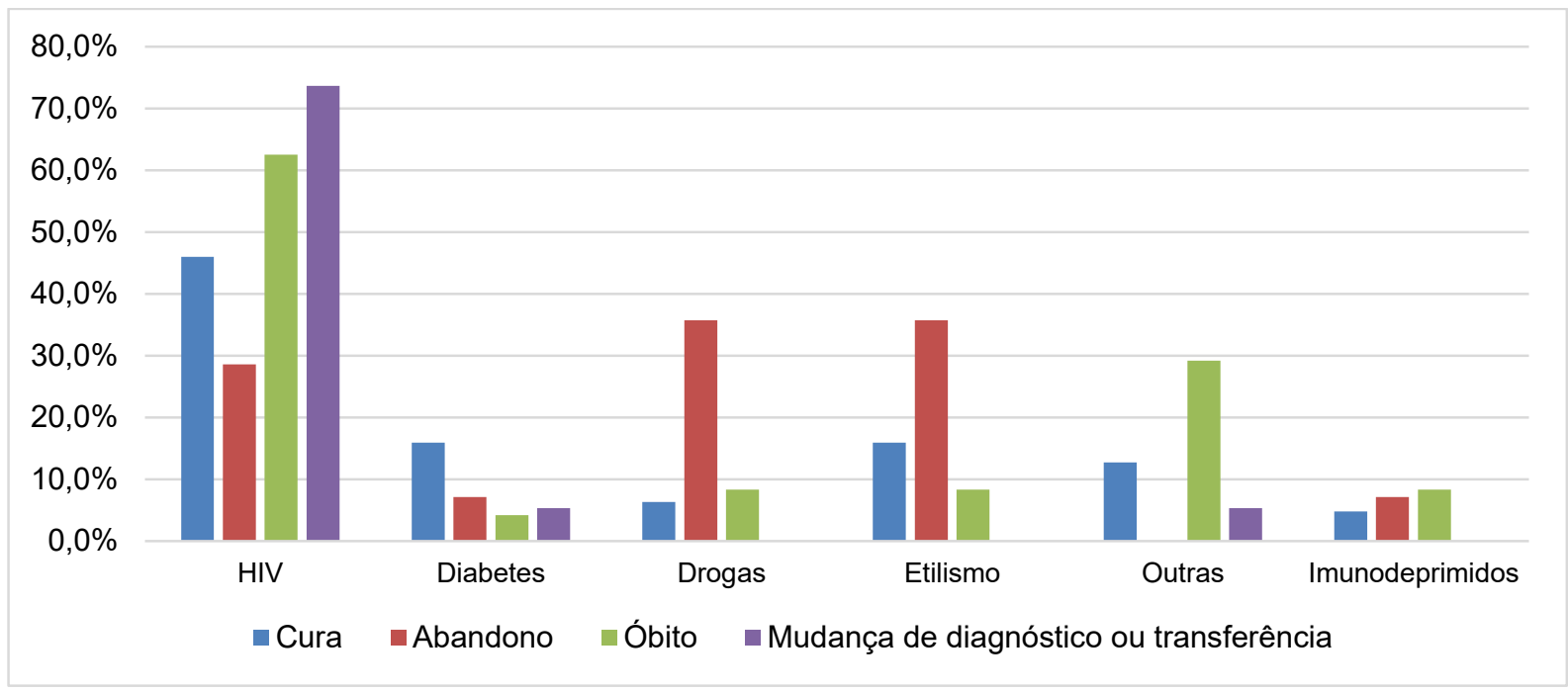

Para os 43 pacientes que tiveram como desfecho de tratamento o óbito, observou-se que $76,7 \%$ eram do sexo masculino, $69,8 \%$ se inseriram na faixa etária entre $20-59$ anos, $51,2 \%$ possuíam até 8 anos de estudo, $88,4 \%$ eram casos novos. Destes pacientes que foram a óbito destaca-se que 31 tinham o registro sobre presença ou ausência de comorbidades, e, $77,4 \%$ apresentaram presença de comorbidades, sendo que desses $62,5 \%$ eram HIV positivo (Figura 1 ).

Em 1979, a Divisão Nacional de Pneumologia Sanitária/Ministério da Saúde (DNPS/MS) normalizou para todo o país um Programa Nacional de Controle da Tuberculose (PNCT) tendo como base fundamental o tratamento quimioterápico, com um esquema de primeira linha, o chamado $2 \mathrm{RHZ} / 4 \mathrm{RH}$, o qual ficou em vigor até outubro de 2009. Uma avaliação do desempenho do PNCT na década de 80 revelou os seguintes resultados do tratamento com o esquema $2 \mathrm{RHZ} / 4 \mathrm{RH}$ em condições de rotina: $79,8 \%$ de cura, com $12,9 \%$ de abandono, $3,3 \%$ de óbito, $2,2 \%$ de falência, $0,2 \%$ de mudança de medicamentos por efeitos colaterais e 1,5\% de transferência, de um total de 145.387 casos analisados. Dos que abandonam, cerca de $30 \%$ retornam positivos ao sistema para retratamento (BRASIL, 1992).

Posteriormente, em 1992, um novo estudo foi realizado a partir de dados de serviços de saúde de oito capitais brasileiras. Esse estudo observou resultados favoráveis, alta por cura comprovada ao completar o tratamento com baciloscopias negativas e alta por cura não comprovada por baciloscopia ao completar o tratamento, em $66 \%$ dos casos de tuberculose pulmonar, enquanto que o percentual de resultados desfavoráveis (abandono, falência e óbito) foi de $34 \%$. Dentre os casos com desfecho desfavorável, a frequência de abandono foi de $26 \%$. Esses achados foram entendidos como uma piora na eficácia do tratamento em relação às coortes de casos analisados na década de 1980(DINIZ, et al, 1995).

Outro estudo realizado no ambulatório de tuberculose do Hospital das Clínicas da Universidade Federal de Pernambuco (UFPE), durante o período de 
agosto de 1994 a dezembro de 1998, analisou o resultado do tratamento da tuberculose pulmonar em 297 doentes. A distribuição dos casos segundo critérios de alta do Hospital das Clínicas da UFPE resultou em: alta por cura comprovada em $54,2 \%$ e alta por cura não comprovada em $24,5 \%$, totalizando um desfecho favorável para o tratamento em $79 \%$ dos casos; a alta por abandono resultou em $12,9 \%$, óbito igual a $4,9 \%$ e falência 3,5\%(ALBUQUERQUE, et al, 2001).

No município de Cuiabá (MT), dos 299 casos novos notificados em 1998, 143 foram bacilíferos, sendo que destes $75,2 \%$ foram curados e $16,1 \%$ abandonaram o tratamento (FERREIRA, 2005).

Já no estudo de casos de tuberculose notificados na Regional Oeste de Belo Horizonte durante o período de 2001 a 2002, e registrados no Sistema de Informação Nacional de Agravos de Notificação (SINAN) observou-se $65,2 \%$ de cura, $12,4 \%$ de abandono do tratamento, $9,6 \%$ de óbitos e $10 \%$ de transferências, mudança de diagnóstico ou dos pacientes em tratamento (PAIXAO; GONTIJO, 2007).

Nos últimos 20 anos, o abandono manteve sempre um índice elevado, com uma média nacional aproximadamente estável de 14\% (ALMEIDA; HONER, 2006). Em 2003, no Brasil, a taxa média de abandono do tratamento situou-se em 12\%; no Estado de São Paulo, foi de 10,3\%, destacando-se enormes diferenças regionais, cujos percentuais encontrados variaram entre 4,5 a $20,3 \%$, sendo $18,7 \%$ a taxa de abandono para Porto Alegre(PAIXAO, GONTIJO, 2007; VIEIRA; RIBEIRO, 2008).Em 2007 o percentual de cura no país, foi de $77,0 \%$ com $6,9 \%$ de abandono (WHO, 2009).

A introdução de etambutol na fase intensiva do tratamento (RHZE, nos dois primeiros meses) é justificada pelo aumento da resistência primária à isoniazida, que migrou de 4,4\% no inquérito nacional de 1995-1997 para 6\% nos anos de 20072008. Cabe ressaltar que a resistência em pacientes hospitalizados ou infectados pelo HIV ainda pode ser maior. Com a introdução de um quarto fármaco, objetivou aumentar o sucesso terapêutico e evitar o aumento de multirresistência (resistência à rifampicina e isoniazida simultaneamente). Além disso, com a introdução da apresentação de dose fixa combinada dos quatro fármacos para a fase intensiva do tratamento, intenta-se aumentar a adesão ao tratamento (BRASIL, 2011c; SANTOS, 2010).

No Brasil e no Rio Grande do Sul, para pacientes em primeiro tratamento, são descritas taxas de aproximadamente de $70 \%$ de cura e $10 \%$ de abandono. Nos casos de retratamento, a taxa de cura cai para pouco mais de $50 \%$ e a de abandono cresce para, aproximadamente, $20 \%$ (BRASIL, 2011 d). No presente estudo, os resultados obtidos para a avaliação do tratamento $2 \mathrm{RHZE} / 4 \mathrm{RH}$, na cidade de Caxias do Sul, foram inferiores às médias descritas na literatura, de $64,5 \%$ de cura, $7,9 \%$ de abandono e $10,2 \%$ de óbito. Estes resultados também não corroboram com as metas preconizadas pela OMS, onde objetiva-se para o controle da doença uma taxa de cura igual ou superior a $85 \%$ e de abandono menor do que $5 \%$ (BRASIL,

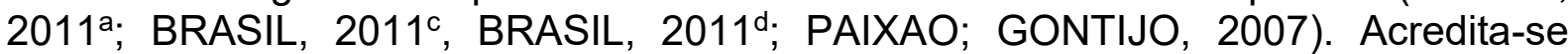
inicialmente que não se tenha atingido os valores de meta em parte em função de 
muitos pacientes serem de retratamento, ou possuírem comorbidades, o que está associado a maior risco de abandono de tratamento. Ainda muitos casos não puderam ser acompanhados até o desfecho em virtude de mudanças de domicílio de pacientes ou mesmo daqueles que, em virtude de seus empregos, residiram temporariamente na região e ali iniciaram tratamento que pode ter sido concluído em outra cidade ou região, o que pode ter causado um aumento de abandono do tratamento e diminuição das taxas de cura aparentes Bases de dados integradas, que permitissem o acesso aos dados de pacientes em todo o país, tornariam possível uma análise mais fidedigna das taxas de abandono nessa população.

Verificamos que a característica do ingresso no tratamento (ser caso novo) e a presença de comorbidades estão relacionadas a baixa adesão estiveram significativamente associados ao abandono do tratamento, e que ser do gênero masculino, estar na faixa etária entre 20-39 anos, possuir baixa escolaridade (até 8 anos de estudo), apresentaram os maiores percentuais de abandono (sem diferença estatística).Nossos dados corroboram com descrito na literatura, que descreve que as principais variáveis que contribuem para o aumento da não adesão e, consequentemente, para o insucesso do tratamento, são de natureza socioeconômica e demográfica, tais como: ser dogênero masculino(FERREIRA, 2005; LIMA, et al, 2001; MENDES; FENTERSEIFER, 2004; PAIXAO; GONTIJO, 2007), estar desempregado ou sem trabalho fixo (BERGEL; GOUVEIA, 2005), possuir baixa escolaridade (BERGEL; GOUVEIA, 2005; PAIXAO; GONTIJO, 2007), o uso frequente de álcool (BERGEL, GOUVEIA, 2005; PAIXAO; GONTIJO, 2007; (RIO GRANDE DO SUL, 2013) e de drogas de abuso (PAIXAO; GONTIJO, 2007; RIO GRANDE DO SUL, 2013), ter realizado tratamento prévio para tuberculose (ALBUQUERQUE, et al, 2001; FERREIRA, 2005), apresentar tuberculose na forma multirresistente (ALBUQUERQUE, et al, 2001), apresentar coinfecção pelo HIV (BRASIL, 2011 ), ter abandonado previamente o tratamento e/ou não ter seu tratamento supervisionado (FERREIRA, 2005). Fatores relacionados ao próprio tratamento farmacológico, como a incidência de efeitos adversos associados ao tratamento (PAIXAO; GONTIJO, 2007), além do o sabor, a quantidade e o tamanho dos comprimidos, influenciam sobre a adesão. Ainda, a baciloscopia negativa no momento do diagnóstico ou durante o tratamento é vista como fator de risco para o abandono do tratamento (BERGEL; GOUVEIA, 2005).

Destaca-se aqui que taxas de abandono são significativamente maiores em pacientes coinfectados pelo HIV, pacientes com baixa escolaridade, pacientes usuários de álcool e/ou de outras substâncias psicoativas e em situações de vulnerabilidade social, como é o caso de moradores de rua (BRASIL, 2011 d). No tocante à coinfecção, a tuberculose representa a primeira causa de morte pessoas que vivem com HIVISIDA (PVHA) no Brasil (BRASIL, 2012; HIJJAR, et al, 2011; PILLER, 2012). Segundo o Relatório Global de Controle da Tuberculose desenvolvido pela OMS, em 2011, as PVHA estão 21 a 34 vezes mais propensas a desenvolver tuberculose ativa quando comparadas à população geral. Frequentemente o diagnóstico da infecção pelo HIV ocorre durante o curso da tuberculose (BRASIL, 2013). O Rio Grande do Sul destaca-se também no cenário nacional com a alta taxa de coinfecção tuberculose/HIV, que gira em torno de $20 \%$ no Estado e $30 \%$ na capital, enquanto que, no Brasil, não passa de $10 \%$. O aumento de casos no Estado a partir de 1992 e a manutenção deles em patamar elevado a 
partir de 2000 ocorreram devido a diversos fatores, como o crescimento de bolsões de misérias nos grandes centros urbanos, a desestruturação dos serviços de saúde e, principalmente, o surgimento da epidemia de SIDA (BRASIL, 2011 d) .Neste estudo, o percentual de coinfecção tuberculose/HIV verificado foi de $27,6 \%$.Tendose em vista que o tratamento da tuberculose é longo (mínimo de seis meses) e que o paciente pode se sentir melhor logo nos primeiro meses, o risco de abandono do tratamento da tuberculose é elevado ao final do primeiro mês e início do segundo, pois corresponde a fase em que os pacientes apresentam-se assintomáticos, com uma boa aparência física e bom estado geral, o que acaba lhes levando à acreditarem que estão livres de doença e por consequência interrompem o tratamento, conforme descrito em estudo realizado por Sá e colaboradores (SÁ, et al, 2007).

Para o aumento da adesão ao tratamento com tuberculostáticos e a diminuição do abandono, é de grande importância garantir que o paciente receba assistência de qualidade em todas as ações relacionadas ao atendimento, o que leva o paciente a reconhecer o serviço e os profissionais que nele atuam como parceiros na recuperação de sua saúde. Além disso, a abordagem individualizada e valorização das atividades educativas propiciam informação decodificada sobre a doença e estimulam o paciente a se tratar. Neste sentido, um trabalho realizado em equipe multidisciplinar pode favorecer e estimular a adesão do paciente_(BOLZAN, CASTILHO, 2008; NEVES, REIS; GIR, 2010; PAIXAO; GONTIJO, 2007).

Em 1997, a OMS passou a recomendar o emprego da estratégia DOTS (Directly Observed Treatment Short course) no tratamento da tuberculose, formalmente oficializada em 1999, por intermédio do Programa Nacional de Controle da Tuberculose (PNCT). Essa estratégia inclui cinco elementos: vontade política, diagnóstico precoce dos casos de tuberculose, aquisição e distribuição regular de medicamentos, tratamento supervisionado (TS) e sistema de informação efetivo. A adoção aumenta a adesão ao tratamento, reduzindo o abandono, elevando as taxas de cura e, portanto, interferindo na transmissão e no risco de desenvolvimento de resistência aos medicamentos tuberculostáticos. O TS consiste em administrar o esquema de tratamento padronizado de curta duração, sob supervisão direta de um observador, durante pelo menos a fase inicial do tratamento a todos os casos de baciloscopias positivas. O TS é uma das prioridades para que o PNCT atinja a meta de curar $85 \%$ dos doentes (BERGEL; GOUVEIA, 2005; BRASIL, 2011 d).

No Brasil, a estratégia DOTS tem sido implantada em vários serviços nos últimos anos. Entretanto, devido a problemas inerentes ao atual sistema público de saúde, como a escassez de recursos humanos e financeiros, há uma dificuldade operacional em empregar este modelo para a maioria dos pacientes que fazem tratamento de tuberculose (BERGEL; GOUVEIA, 2005). 


\section{CONSIDERAÇÕES FINAIS}

Destaca-se que os esquemas terapêuticos avaliados nesse estudo (2RHZ/4RH e 2RHZE/4RH) não atingiram as metas da OMS para o controle da tuberculose, no mínimo $85 \%$ de cura e no máximo $5 \%$ de abandono. Dentre os fatores preditivos do abandono ao tratamento foi verificado que o gênero masculino, a faixa etária entre 20-39 anos, a baixa escolaridade (até 8 anos de estudo), caso novo e presença de comorbidades apresentaram os maiores percentuais, e, desses fatores estiveram significantemente associados ao desfecho do tratamento: ingresso (caso novo) e presença de comorbidades.

Para o controle da tuberculose a diminuição dos índices de abandono ao tratamento se faz importante, pois o abandono tornou-se um dos principais desafios para o sistema de saúde brasileiro acarretando aumento da infectividade e transmissão da doença, recidiva, desenvolvimento de resistência aos tuberculostáticos e à morte. Estratégias para a redução do índice de abandono ao tratamento devem ser traçadas, visando à atuação de uma equipe multidisciplinar que preste assistência qualificada. Além disso, a organização de grupos educativos focando o acolhimento dos pacientes também é uma estratégia que deve ser colocada em prática, onde a informação adequada sobre a doença seja difundida para os pacientes e seus familiares. O melhor recurso disponível para o controle da tuberculose no sistema de saúde continua sendo a detecção precoce e o tratamento correto.

\section{REFERÊNCIAS}

ALBUQUERQUE, M.F.M.; et al. Fatores prognósticos para o desfecho do tratamento da tuberculose pulmonar em Recife, Pernambuco, Brasil. Pan Am J Public Health, v. 9, n. 6, p. $368-74.2001$.

ALMEIDA, A.S.; HONER, M.R. Abandono do tratamento da tuberculose em duas unidades de referência de Campo Grande, MS - 2002 e 2003. Bol Pneumol Sanit, v. 14, n. 3, p. 167-171, 2006.

BERGEL, F.S.; GOUVEIA, N. Retornos frequentes como nova estratégia para adesão ao tratamento de tuberculose. Rev Saúde Pública. v. 39, n. 6, p. 898-905. 2005.

BOLZAN, M.H.; CASTILHO, S.R. Intervenções farmacêuticas para uso racional de medicamentos. Escola de Saúde do Exército. Programa de Pós-Graduação em Aplicações Complementares às Ciências Militares. Rio de Janeiro; 2008. 
BRASIL. Ministério da Saúde. FUNASA/PNCT. Documento básico da reunião de avaliação operacional e epidemiológica do PNCT na década de 80. Brasília: MS, 1992.

. Guia de vigilância epidemiológica. 7.ed. Brasília: MS, Secretaria de Vigilância em Saúde, Departamento de Vigilância Epidemiológica, 2009a.

. Programa Nacional de Controle da Tuberculose. Nota técnica sobre as mudanças no tratamento da tuberculose no Brasil para adultos e adolescentes - versão 2. Brasília: MS. 2009 .

. Manual de Recomendações para o Controle da Tuberculose no Brasil. Programa Nacional de Controle da Tuberculose. Brasília: MS. 2010.

Manual de recomendações para o controle da tuberculose no Brasil. Brasília: MS, Saúde, Secretaria de Vigilância em Saúde, Departamento de Vigilância Epidemiológica, 2011․

. Programa Nacional de Controle da Tuberculose. Situação da tuberculose no Brasil. Brasília: MS, Conselho Nacional de Saúde, 2011 ${ }^{\text {b }}$

. Tratamento diretamente observado (TDO) da tuberculose na atenção básica: protocolo de enfermagem. Brasília: MS, Secretaria de Vigilância em Saúde, Departamento de Vigilância Epidemiológica, 2011'.

. Tuberculose na Atenção Primária à Saúde. 2.ed. Brasília: MS, Secretaria de Atenção à Saúde. Departamento de Atenção Básica, 2011 .

Boletim Epidemiológico. v. 43, Especial Tuberculose. Brasília: Secretaria de Vigilância em Saúde, 2012.

Recomendações para o manejo da coinfecção TB-HIV em serviços de atenção especializada a pessoas vivendo com HIVIAIDS. Brasília: MS, Secretaria-Executiva, 2013.

CHIRINOS, N.E.C.; MEIRELLES, B.H.S. Fatores associados ao abandono do tratamento da tuberculose: uma revisão integrativa. Texto Contexto Enferm, Florianópolis 2011; 20(3):599-606.

DINIZ, L.S.; et al. Efetividade do tratamento da tuberculose em oito municípios e capitais brasileiras. Bol Pneumol Sanitaria (Rio de Janeiro), v. 3, n. 1, p. 6-18. 1995.

DUCATI, R.G.; BASSO, L.A.; SANTOS, D.S. Micobactérias. In: TRABULSI, L.R.; ALTERTHUM, F. (Eds.). Microbiologia. 5.ed. São Paulo: Atheneu, 2008. p. 423-31.

FERREIRA, S.M.B.; SILVA, A.M.C.; BOTELHO, C. Abandono do tratamento da tuberculose pulmonar em Cuiabá - MT - Brasil. J Bras Pneumol, v. 31, n. 5, p. 42735, 2005. 
HIJJAR, M.A.; et al. Epidemiologia da tuberculose: importância no mundo, no Brasil e no Rio de Janeiro. Curso da tuberculose. Pulmão, Rio de Janeiro, v. 14, n. 4, p. 310-14. 2005.

LIMA, M.B.; et al. Estudo de casos sobre abandono do tratamento da tuberculose: avaliação do atendimento, percepção e conhecimentos sobre a doença na perspectiva dos clientes (Fortaleza, Ceará, Brasil). Cad Saúde Pública, v. 17, n. 4, p. 877-85, 2001.

MENDES, A.M.; FENSTERSEIFER, L.M. Tuberculose: por que os pacientes abandonam o tratamento? Bol Pneumol Sanit, v. 12, n. 1, p. 25-36, 2004.

MIMS, C.; et al. Microbiologia médica. 2.ed. São Paulo: Manole, 1999.

NEVES, L.A.S.; REIS, R.K.; GIR, E. Adesão ao tratamento por indivíduos com a coinfecção HIV/tuberculose: revisão integrativa da literatura. Revista Esc Enferm USP, v. 44, n. 4, p. 1135-41, 2010.

PAIVA, V.S.; PEREIRA, M.; MOREIRA, J.S. Perfil epidemiológico da tuberculose pulmonar em Unidade Sanitária de referência em Porto Alegre, RS. Rev AMRIGS, v. 55, n. 2, p. 113-17, 2011.

PAIXÃO, L.M.M.; GONTIJO, E.D. Perfil de casos de tuberculose notificados e fatores associados ao abandono, Belo Horizonte, MG. Rev Saúde Pública, v. 41, n. 2, p. 205-13, 2007.

PILLER, R.V.B. Epidemiologia da Tuberculose. Pulmao, Rio de Janeiro, v. 21, n. 1, p. 4-9, 2012.

RIO GRANDE DO SUL. Plano Estadual de Saúde: 2012/2015. Grupo de Trabalho Planejamento, Monitoramento e Avaliação da Gestão (Org.). Porto Alegre:

Secretaria da Saúde; 2013.

SÁ, L.D; et. al. Tratamento da tuberculose em unidades de saúde da família: histórias de abandono. Texto Contexto Enferm, v. 16, n. 4, p. 712-718, 2007.

SANTOS, R.P. Antituberculosos. In: FUCHS, F.D.; WANNMACHER, L. (Eds.).

Farmacologia Clínica: fundamentos da terapêutica racional. 4 ed. Rio de Janeiro: Guanabara Koogan, 2010. p. 544-54.

SANTOS, Y.S.M.F. Assistência farmacêutica na tuberculose. Instituto Salus, 2012, p. 1-9.

SBPT. Sociedade Brasileira de Pneumologia e Tisiologia. Comissão de Tuberculose. III Diretrizes para tuberculose da SBPT. J Bras Pneumol, v. 35, n. 10, p. 1018-1048 2009.

SOUZA, M.V.N.; VASCONCELOS, T.R.A. Fármacos no combate à tuberculose: passado, presente e futuro. Quim Nova, v. 28, n. 4, p. 678-82, 2005. 
VIEIRA, A.A.; RIBEIRO, S.A. Abandono do tratamento de tuberculose utilizando-se as estratégias tratamento auto-administrado ou tratamento supervisionado no

Programa Municipal de Carapicuíba, São Paulo, Brasil. J Bras Pneumol, v. 34, n. 3, p. 159-66, 2008.

VILLA, T.C.S.; et al. Fatores preditivos aos resultados desfavoráveis no tratamento da tuberculose: revisão integrativa da literatura (2001-2005). Braz J Nurs, 2008.

WHO. World Health Organization. Global Tuberculosis Control Report. Genebra: WHO; 2011. $\overline{\mathrm{WHO}} 2009$.

Global Tuberculosis Control: epidemiology, strategy, financing. Genebra:

Artigo recebido em: 21/10/2016

Artigo aprovado em: 23/10/2017

Artigo publicado em: 20/12/2017 